\title{
Effects of Pleurotus ostreatus on Blood Pressure and Glycemic Status of Hypertensive Diabetic Male VolunteersS
}

\author{
MBK Choudhury ${ }^{1}$, T Rahman $^{2}$, AJ Kakon ${ }^{2}, \mathrm{~N} \mathrm{Hoque}^{3}, \mathrm{M} \mathrm{Akhtaruzzaman}^{4}$, \\ MM Begum ${ }^{5}$, MSK Choudhuri ${ }^{6}$, MS Hossain ${ }^{7}$ \\ ${ }^{1}$ Directorate General Health Services, Dhaka, ${ }^{2}$ National Mushroom Development and Extension Centre \\ (NAMDEC), Savar, Dhaka, ${ }^{3}$ Dept of Biochemistry, Faridpur Medical College, Faridpur \\ ${ }^{4}$ Dept of Biochemistry, Shaheed Suhrawardy Medical College, Dhaka, ${ }^{5}$ Dept of Physiology, Shaheed \\ Suhrawardy Medical College, Dhaka, ${ }^{6}$ Dept of Pharmacy, Jahangirnagar University, Dhaka \\ ${ }^{7}$ Dept of Biochemistry and Molecular Biology, Jahangirnagar University, Dhak
}

\begin{abstract}
Different species of mushrooms are being used increasingly for their nutritional and medicinal values. They are used in traditional medical practice in the treatment and prevention of diabetes, obesity, heart diesaes, hyperacidity, cancer and hypertension. This study was designed to see the effects of Pleurotus ostreatus on blood pressure and glycemic status of hypertensive diabetic male volunteers. The study was carried out in the National Mushroom development and Extension Center (NAMDEC) laboratory, Savar, Dhaka in collaboration with the Department of Pharmacy along with the Department of Biochemistry and Molecular Biology, Jahangirnagar University. A total of 27 hypertensive male volunteers suffering from type 2 diabetes mellitus with age range of 32 to 68 years, who were free from renal impairment and other known acute or chronic diseases were included in the study. Blood pressure, fasting plasma glucose, HbAlc and serum creatinine were measured before and after 3 months of regular intake of 3 grams mushroom powder capsule daily in in 3 divided doses. However they were allowed to continue the drugs they were taking already. The study showed that after 3 months of regular intake of Pleurotus ostreatus mushroom, both systolic and diastolic blood pressure decreased significantly $(\mathrm{p}<0.001)$. It was also observed that, Pleurotus ostreatus decreased fasting plasma glucose level significantly $(\mathrm{p}<0.001)$. Reduction of $\mathrm{HbA} 1 \mathrm{c} \%$ observed after 3 months of mushroom intake was found to be significant $(p<0.001)$. But there was no significant change of plasma creatinine level $(\mathrm{p}>0.05)$ indicating Pleurotus ostreatus has no detrimental effect on renal system. From the study, it can be said that, Pleurotus ostreatus mushroom intake improves glycemic status and blood pressure control in diabetic hypertensive subjects.

Key words: Pleurotus ostreatus, Hypertension, Diabetes Mellitus, $\mathrm{HbA}_{1 \mathrm{c}}$
\end{abstract}

\section{Introduction}

Health is a birth right of every individual. There is increasing evidence that the dietary habits of people are important determinants of health. Proper dietary substances can protect people from chronic diseases such as coronary heart disease, cancer, obesity and diabetes mellitus. Diabetes mellitus is a universal health problem affecting human society at all stages of development ${ }^{1}$. It is primarily caused by degeneration and inactivation of the $\beta$ cells of islets of langerhans of the pancreas. Serious defect of carbohydrate, fat and protein metabolism occurs in this disorder ${ }^{2}$. Increasing evidence in both experimental and clinical studies suggests that, there is a close link between hyperglycemia and diabetic complications ${ }^{3}$. 
Diabetes is a condition than can usually be controlled by proper dietary management. Recent studies indicated that foods with low glycemic index has a significant influence on blood glucose levels. Chemical and biochemical hypoglycemic agents, e.g. insulin, tolbutamide, phenformin, troglitazone, rosiglitazone and repaglinides are the mainstay of treatment of diabetes and are effective in controlling hyperglycemia but they are not free from harmful side-effects and fail to significantly alter the course of diabetic complications. Traditional medicines such as mushrooms are increasingly being used for treatment of certain health problems. Some mushrooms appear to be effective for both the control of blood glucose level and the modification of the course of diabetic complications without side-effects ${ }^{4}$.

Mushrooms are nutritive and are richer in protein than cereals, pulses, fruits and vegetables on dry weight basis 5 . Due to their low caloric value, mushrooms can be consumed by patients with hyperlipidemia ${ }^{6}$. They are completely devoid of starch and are an excellent inclusion in the diet of diabetic patients. Edible fungi produce secondary metabolites which possess various therapeutic properties. Mushrooms also contain ample minerals such as calcium, phosphorous, potassium, iron and copper. They have traditionally been used in the treatment and prevention of diabetes, obesity, heart disease, hyperacidity, constipation, cancer and hypertension ${ }^{7}$. Mushrooms are very useful in the prevention of diabetes mellitus due to its polysaccharides with low glycemic index and because it is devoid of sugar and starch.

Mushrooms are edible fungi which have been used as an antidiabetic drug since ancient times. Pleurotus ostreatus, the Oyster mushroom, is increasingly being recognized as an important food product with a significant role in human health and nutrition ${ }^{8}$. Pleurotus species are rich sources of proteins, minerals $(\mathrm{Ca}, \mathrm{P}, \mathrm{Fe}, \mathrm{K}$ and $\mathrm{Na}$ ), vitamin $\mathrm{C}$ and vitamin $\mathrm{B}$ complexes (thiamine, riboflavin, folic acid and niacin)9. Pleurotus species contains high potassium to sodium ratio, which makes mushrooms an ideal food for patients suffering from hypertension and heart diseases. Treatment with mushroom, Pleurotus ostreatus extracts (especially high level) showed to reduce the high blood glucose level in hyperglycemic rats ${ }^{10}$. Pleurotus species also possess blood-pressure-lowering activity. Recent studies showed that, P. cornucopiae exhibit antihypertensive activity which might be attribured to their D-mannitol contents, which inhibits angiotensin converting enzyme. P. ostreatus has been found to contain highest amount of lovastatin in the fruit-body, especially in the lamellae or gills ${ }^{11}$.

Hypertension or high blood pressure, sometimes called arterial hypertension, is a chronic medical condition in which the blood pressure in the arteries is elevated. This requires the heart to work harder than normal to circulate blood through the blood vessels. Hypertension is a major risk factor for stroke, myocardial infarction (heart attacks), heart failure, aneurysms of the arteries (e.g. aortic aneurysm), peripheral arterial disease and is a cause of chronic kidney diseases. Even moderate elevation of arterial blood pressure is associated with a shortened life expectancy. Dietary and life style changes can improve blood pressure control and decrease the risk of associated health complications, although drug treatment is often necessary in people for whom lifestyle changes prove ineffective or insufficient.

Pleurotus ostreatus produces significant increases in liver glycogen when compared to diabetic controls and it was suggested that increase in liver glycogen may be due to enhanced rate of glycogenesis ${ }^{12}$. In a study, it was observed that Pleurotus ostreatus at the dose of $100 \mathrm{mg} / \mathrm{kg} /$ day is the most suitable one in reducing blood glucose and lipid levels ${ }^{13}$, which ultimately can improve hypertension.

Diabetes, dyslipidemia and hypertension runs parallely in relation to mortality and morbidity. But there is no easy procedure to overcome them. Recently, mushroom have become the target of studies trying to determine if fungi 
have nutritional and medicinal benefits which could improve the health status of diabetic subjects specially suffering from hypertension.

\section{Materials and Methods}

The study was conducted in the Laboratory of Strengthening Mushroom Development Project, National Mushroom Development and Extension Center (NAMDEC), Sobhanbag, Savar, Dhaka. A total of 27 hypertensive male volunteers suffering from from type 2 diabetes with age range of 32 to 68 years, who were free from renal impairement and other known acute and chronic diseases residing at Savar (Dhaka) area were included in the study after taking their informed written consent. Subjects with a history of addiction other than smoking were also excluded from the study. They were allowed to continue the medication they were taking. Age, sex, occupation, educational status, marital status, family history and drug history were recorded in a preformed data collection sheet.

At the beginning of the study, subjects were evaluated for health status. Both systolic and diastolic blood pressure were measured following standard procedure using sphyg momanometer by a trained physician following standard procedure. Mean of duplicate measurements was taken. With all aseptic precautions, $10 \mathrm{ml}$ of fasting blood was collected from median cubital vein. Immediately after collection, blood was poured into fluoride and EDTA containing test tube. The test tube was then gently shaken for proper mixing with the anticoagulants. $1 \mathrm{ml}$ of this anticoagulantmixed whole blood was taken in an eppendorf for estimation of $\mathrm{HbAlc}_{1}$. The remaining blood was centrifuged at $3000 \mathrm{rpm}$ for 5 minutes. Separated plasma was transferred into two eppendorf containing $1 \mathrm{ml}$ in each. Plasma crearinine was estimated by alkaline picrate method. Plasma glucose level was estimated by enzymatic 'Glucose oxidase' method. Analysis was done by semi auto biochemical analyzer 3000 evaluation using commercially available reagent kit. Glycated hemoglobin was estimated by a photometric method using 'Stanbio reagent kit'. All the tests were carried out as early as possible.

Fresh fruiting bodies of Pleurotus ostreatus were collected from culture house of National Mushroom Development and Extension Centre (NAMDEC). Collected mushrooms were then sun dried at moisture level $4-5 \%$, then grinded and poured into capsule shells which contain 500 mg powder. Prepared capsules were ready to dispense and preserved into moisture free glass containers.

Mushroom capsules containing $500 \mathrm{mg}$ Pleurotus ostreatus powder in each were supplied to take two capsules three times daily, so that each subject took $3 \mathrm{gms}$ mushroom powder daily.

After three months the subjects were reevaluated and all the laboratory investigations were repeated.

Results were expressed as mean \pm SE. Paired Student's ' $t$ ' test was used to see the level of significance. $95 \%$ confidence limit was taken as level of significance.

\section{Reasult}

Mean $( \pm \mathrm{SE})$ serum creatinine $(\mathrm{mg} / \mathrm{dl})$ level before and after three months of mushroom supplementation were $0.92 \pm 0.05$ and $1.01 \pm 0.04$ respectively (Fig. 1). No statistically significant mean difference of serum creatinine between the two periods was observed $(\mathrm{p}>0.05)$.

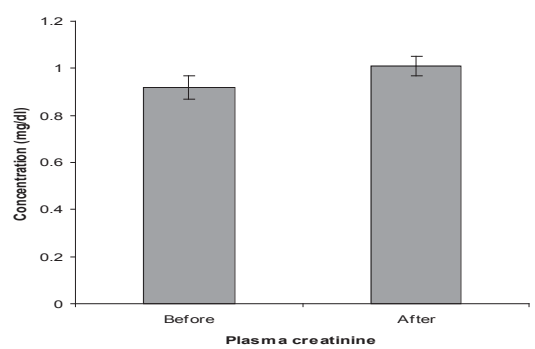

Fig. 1: Plasma creatinine (mean \pm SE) 
The Mean $( \pm \mathrm{SE})$ systolic blood pressure $(\mathrm{mmHg})$ before and after three months of mushroom treatment were $148.68 \pm 4.20$ and $133.42 \pm 3.62$ respectively (Fig. 2). A highly significant mean difference of systolic blood pressure between the two periods was observed $(\mathrm{p}<0.001)$. The mean $( \pm \mathrm{SE})$ of diastolic blood pressure $(\mathrm{mmHg})$, before and after mushroom supplementation were $90.00 \pm 1.98$ and $80.00 \pm 2.44$ respectively (Fig. 2) which was significant statistically $(\mathrm{p}<0.001)$.

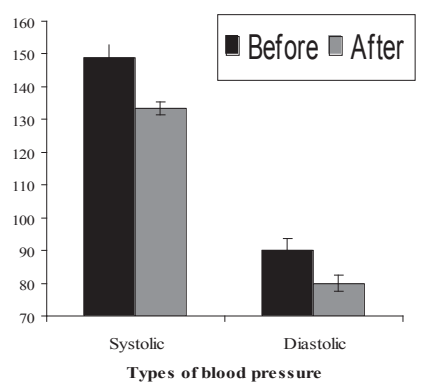

Fig. 2: Systolic and diastolic blood pressure (mean \pm SE)

The Mean $( \pm$ SE) fasting plasma glucose level (mmol/L) before mushroom treatment was $10.36 \pm 0.72$ which was reduced to $8.48 \pm 0.58$ after three months of mushroom treatment (Fig. 3). A highly significant mean difference of plasma glucose between the two periods was observed $(\mathrm{p}<0.001)$. Mean $( \pm \mathrm{SE})$ of HbA1c (\%), were found to be $8.2 \pm 0.36$ and $7.1 \pm 0.31$ before and after mushroom treatment respectively (Fig. 3). A highly significant mean difference of $\mathrm{HbAlc} \%$ observed between the two periods $(\mathrm{p}<0.001)$.

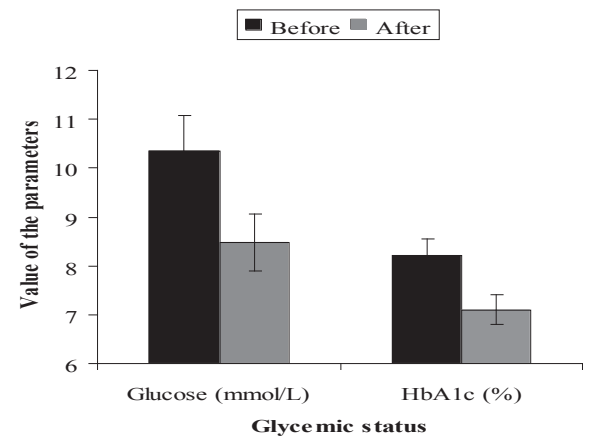

Fig. 3: Glycemic status o the subjects $($ mean \pm SE)

\section{Discussion}

Oyster mushroom (Pleurotus sp) is known in the Indian traditional system of medicine for its antihyperglycemic and antihyperlipidemic potential. Mushrooms are edible fungi confirmed to have definite benefit in human health and nutrition. Oyster mushrooms have been demonstrated to have beneficial effects in animal and human studies individually as well as in combination. However, the effect of Oyster mushroom on high blood glucose levels in diabetes was not clear in previous studies. Hence, the present study was undertaken to observe the effect of Oyster mushroom (Pleurotus ostreatus) on blood pressure and glycemic status of hypertensive diabetic male volunteers.

No significant variation of serum creatinine level of the study subjects before and after three months of supplementation of mushroom capsules (3 gms/day), indicates that Oyster mushroom has no detrimental effect on kidney.

In this study, it was observed that both systolic and diastolic blood pressure decreased significantly after three months of mushroom treatment. Findings of previous animal trial support this observation. In a study, Talpur14 observed reduction of both systolic and diastolic blood pressure significantly in diabetic Zucker fatty rats with mushroom supplements and maintained body weight compared with control animals. Another study concluded that, the soluble fraction of Maitake mushroom lowers systolic blood pressure significantly ${ }^{15}$. Although there is no sufficient human data, it was reported that mushrooms significantly reduces systolic and diastolic blood pressure in diabetic subjects ${ }^{16}$. In another study the author observed reduction of blood pressure (systolic and diastolic) of hypertensive subjects significantly ${ }^{17}$. Mushrooms have been found to ameliorate hypertension via an effect on systolic blood pressure that may, at least in part, involve the renin-angiotensin system ${ }^{18}$. Now a days, it is strongly suggested that Oyster mushroom contains statins which reduces plasma cholesterol, triglyceride and phospholipids. 
MBK Choudhury, T Rahman, AJ Kakon, N Hoque et al

Some authors demonstrated that, addition of statins led to a greater reduction in systolic blood pressure (SBP), mean arterial pressure (MAP) and diastolic blood pressure (DBP) ${ }^{19,20}$. Collectively, these suggest that statins may be more effective in lowering blood pressure levels when added to antihypertensive drugs.

Cholesterol lowering is an alternative therapy that may potentially target arterial stiffness and thus blood pressure, through its effects on endothelial function and arterial wall composition. A number of studies in hypercholesterolemic patients have shown improvement, particularly in peripheral artery properties, with cholesterol-lowering therapy ${ }^{21-23}$. It is not clear whether a cholesterol reduction within the normal clinical range in patients with isolated systolic hypertension (ISH) might also reduce stiffness of the large arteries, and thereby reduces systolic blood pressure (SBP). Our current study is in agreement with these findings.

Reactive oxygen species have been reported to be important in ischemic reperfusion injury cascades which are important factors for hypertension. In a study Mowsumi et al. ${ }^{24}$ demonstrated that Calocybe indica and Pleurotus djamor mushroom extracts are capable of scavenging free radicals. These observations indicate that Oyster mushroom is able to improve both systolic and diastolic blood pressure by their free radical scavenging activities.

Our study showed that both fasting plasma glucose and HbA1c \% of hypertensive diabetic subjects were reduced significantly after three months supplementation of a considerable amount (3 grams per day) of dried P. ostreatus capsules. The significant fall in fasting plasma glucose and $\mathrm{HbA} 1 \mathrm{c} \%$ may be attributed to the hypoglycemic potential of the Oyster mushroom supplement. It was reported that mushroom significantly reduces blood glucose level in diabetic subjects ${ }^{16,17}$. Reduction in glycated hemoglobin in streptozotocin induced diabetic mice after mushroom supplementation was also observed $^{25}$. Treatment with mushroom Pleurotus

ostreatus extract (especially high level) can reduces the high blood glucose level in hyperglycemic $\operatorname{rats}^{10}$. Our findings are in agreement with all of these findings.

The Pleurotus species of Oyster mushroom have been proven to be a good source of almost all essential amino acids that have many medicinal properties and antioxidant activities ${ }^{26,27}$. In a study, antihyperglycemic effects were demonestrated with a water soluble polysaccharide from $P$. citrinopileatus fermentation broth. The polysaccharide was effective in lowering blood glucose levels in diabetic rats $^{28}$. All of these findings support antihyperglycemic activities of Pleurotus ostreatus which are also supportive of our findings.

\section{References}

1. Rai RD, Sohi. How protein rich are mushrooms. Indian Hort 1998; 33: 2-3

2. Bahl N. Food value of mushrooms. New Delhi: Oxford \& IBH publishing 2000.

3. Rabbani SI, Devi K, Khanam S. Inhibitory effect of glimepiride on nicotinamide - streptozotocin induced nuclear damage and sperm abnormality in diabetic Wister Rats. Ind J Exp Biol 2009; 47: 804-810.

4. Perera PK, Li Y. Mushrooms as a functional food mediator in Preventing and ameliorating diabetes. Functional Foods in Health and Disease 2011; 4: 161-171.

5. Ghosh C. Nutritional value of edible mushroom. In the biology and cultivation of edible mushroom. New York: Academic press 1990.

6. Bano Z. Pleurotus mushroom as nutritional food. Tropical mushroom - biological nature and cultivation method. Hong Kong: The Chinese University Press 1982.

7. Suguna S, Usha M. Cultivation of oyster mushroom. J food sci technol 1995; 32: 351-352.

8. Alam N, Amin R, Khan A, Ara I, Shim MJ, Lee MW, Lee TS. Nutritional analysis of cultivated mushrooms in Bangladesh: Pleurotus ostreatus, Pleurotus sajor-caju, Pleurotus florida and Calocybe indica. Mycobiology 2008; 36: 228-232.

9. Ça?lar?rmak N. The nutrients of exotic mushrooms (Lentinula edodes and Pleurotus species) and an estimated approach to the volatile compounds. Food Chemistry 2007; 105: 1188-1194. 
Effects of Pleurotus ostreatus on Blood Pressure and Glycemic

10. Ghaly IS, Ahmed ES, Booles HF, Farag IM, Nada SA. Evaluation of antihyperglycemic action of Oyster mushroom (Pleurotus ostreatus) and its effect on DNA damage, chromosome aberrations and sperm abnormalities in streptozotocin-induced diabetic rats. Global Veterinaria 2011; 7: 532-544.

11. Gunde-Cimerman N, Cimerman A. Pleurotus fruiting-bodies contain the inhibitor of 3-hydroxy-3methylglutaryl-Coenzyme A reductase-lovastatin. Experimental Mycology 1995; 19: 1-6.

12. John P. M.V.Sc. Thesis, Kerala Agricultural University, Thrissur 2003; p 62.

13. Krishna LS, Usha PTA, Nair AMC. Hypoglycaemic effect of pleurotus ostreatus in rats. Indian J Anim Res 2009; 43: 139-141.

14. Talpur N, Echard BW, Yashmin T, Bagchi D, Preuss HG. Effects of niacin-bound chromium, Maitake mushroom fraction SX and (-)hydroxycitric acid on the metabolic syndrome in aged diabetic Zucker fatty rats. Mol Cell Biochem 2003; 252: 1-2,369-377.

15. Talpur N, Echard BW, Dadgar A, Aggarwal S, Zhuang C, Bagchi D, Preuss HG. Effects of Maitake mushroom fractions on blood pressure of Zucker fatty rats. Res Commun Mol Pathol Parmacol 2002; 112:1-4,68-82.

16. Khatun K, Mahtab H, Khanam PA, Sayeed MA, Khan KA. Oyster mushroom reduced blood glucose and cholesterol in diabetic subjects. Mymensingh Med J 2007;16:94-99.

17. Choudhury BK, Amin SMR, Sarkar NC, Khan AS, Mahjabin T, Begum R, Akhtaruzzaman M, Rahman MS. Impact of oyster mushroom (Pleurotus ostreatus) intake on hypertension and blood sugar status of common people of Bangladesh. Bangladesh J Med Biochem 2008;1(1):14-17.

18. Preuss HG, Echard B, Bagchi D, Perricone NV. Maitake Mushroom Extracts Ameliorate Progressive Hypertension and Other Chronic Metabolic Perturbations in Aging Female Rats. Int J Med Sci 2010; 7: 169-180.

19. Borghi C, Prandin MG, Costa FV, Bacchelli S, Degli Esposti D, Ambrosioni E. Use of statins and blood pressure control in treated hypertensive patients with hypercholesterolemia. J Cardiovasc Pharmacol 2000; 35: 549-555.
20. Sposito AC, Mansur AP, Coelho OR, Nicolau JC, Ramires JA. Additional reduction in blood pressure after cholesterol-lowering treatment by statins (lovastatin or pravastatin) in hypercholesterolemic patients using angiotensin-converting enzyme inhibitors (enalapril or lisinopril). Am J Cardiol 1999; 83:1497-1499.

21. Smilde TJ, van den Berkmortel FW, Wollersheim $\mathrm{H}$, van Langen H, Kastelein JJ, Stalenhoef AF. The effect of cholesterol lowering on carotid and femoral artery wall stiffness and thickness in patients with familial hypercholesterolaemia. Eur J Clin Invest 2000; 30: 473-480.

22. 13. Matthews PG, Wahlqvist ML, Marks SJ, Myers KA, Hodgson JM. Improvement in arterial stiffness during hypolipidaemic therapy is offset by weight gain. Int J Obes Relat Metab Disord 1993; 17:579-583.

23. Yasuaki T, Fumio O, Nobuaki T, Yuichiro W, Ikuo T, Shumpei A, Chieko M, Shiro O, Kazuyoshi O, Masunori M. Improvement of atherosclerosis and stiffness of the thoracic descending aorta with cholesterollowering therapies in familial hypercholesterolemia. Arterioscler Thromb Vasc Biol 1996; 16: 955-962.

24. Mowsumi FR, Rahaman A, Choudhury MBK, Sarker NC, Hossain S. Comperative in vitro Free Redical Scavenging Effects of Calocybe indica and Pleurotus djamor. Bangladesh J Mushroom 2011; 5: 9-15.

25. Swanston-Flatt SK, Day C, Flatt PR, Gould BJ, Bailey CJ. Glycaemic effects of traditional European plant treatments for diabetes. Studies in normal and streptozotocin diabetic mice. Diabetes Res 1989; 10; 69-73.

26. 23. Jayakumar T, Ramesh E, Geraldine P. Antioxidant activity of the oyster mushroom, Pleurotus ostreatus, on $\mathrm{CCl}$-induced liver injury in rats. Food and Chemical Toxicol 2006; 44: 1989-1996.

27. 37. Mattila $P$, Salo-Väänänen $P$, Könkö K, Aro H, Jalava T. Basic composition and amino acid contents of mushrooms cultivated in Finland. J Agric Food Chem 2002;50:6419-6422.

28. Hu SH, Liang ZC, Chia YC, Lien JL, Chen KS, Lee MY, Wang JC. 2006. Antihyperlipidemic and antioxidant effects of extracts from Pleurotus citrinopileatus. J Agric Food Chem 2006; 54: 2103-2110. 